\title{
Marginal Integrity of CAD/CAM Fixed Partial Dentures
}

\author{
Martin Rosentritta, MS \\ Michael Behrb, DDS, PhD \\ Carola Kolbeck', DDS \\ Gerhard Handeld, DDS, MS, PhD
}

\section{ABSTRACT}

Objectives: Computer-aided design (CAD) and manufacturing (CAM) allows the milling of high strength zirconia fixed partial dentures (FPD), however bonding to an inert $\mathrm{ZrO}_{2}$ ceramic surface may effect the marginal integrity of the FPDs. The aim of this investigation was to evaluate the marginal adaptation of zirconia FPDs at the interfaces between zirconia, cement, and tooth.

Methods: 32 3-unit FPDs were fabricated of the CAD/CAM Y-TZP zirconia (Lava, 3M Espe, Germany) according to the manufacturers' instructions. Resin cements with corresponding primer and bonding systems were used to lute the FPDs: Compolute/EBS multi (3M Espe, Germany). Panavia F/ED (Kuraray, Japan), Variolink 2/Syntac classic (Ivoclar-Vivadent, FL) and RelyX Unicem/without treatment (3M Espe, Germany). Au-alloy FPDs (BioPontostar, Bego, Germany) were cemented with RelyX Unicem and Harvard (Harvard, Germany) as the control. Marginal adaptation was evaluated with scanning electron microscopy using replica specimen before and after artificial aging. After aging, microleakage tests were performed with fuchsine solution. The interfaces between cement-tooth and cement-FPD were examined.

Results: At the interfaces (cement-tooth and cement-FPD), the systems showed a 95\% or higher perfect margin before and after aging. Only Variolink2/Syntac had a marginal adaptation, lower than a $70 \%$ perfect margin. Generally, the fuchsine penetration was below $20 \%$, only BioPontstar/Harvard and Lava/Variolink2 showed penetration results between $80 \%$ and $100 \%$.

Conclusion: The success of the adhesive cementation of zirconia FPDs depends on the cement system. Under the conditions of this study, zirconia FPDs showed good to sufficient marginal integrity in combination with Panavia/ED, Compolute/EBS and RelyX Unicem. (Eur J Dent 2007;1:25-30)
\end{abstract}

Key words: CAD/CAM; Marginal integrity; Microleakage; Zirconia.

\section{INTRODUCTION}

Pressable all-ceramic materials exhibit a fracture resistance which limits the application of the materials to full coverage single unit restorations. The use of high strength hot isostatic pressed (hipped) or partly stabilized zirconia ceramics in combination with computer aided manufacturing

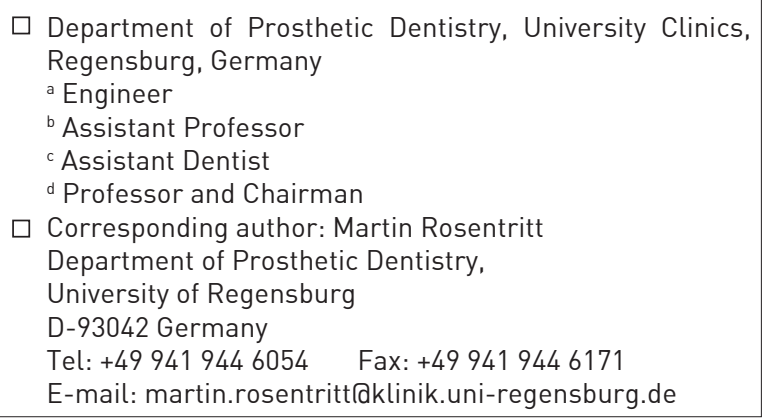

(CAM) represents an effort to increase the indications in which all-ceramics can be used to replace posterior teeth. The concepts extend from manufacturing "green" or "white" pre-sintered ceramic cores (Cercon, DeguDent; Lava, 3M Espe) to the milling of high strength hipped zirconia cores (Digizon, Girrbach). While all systems use CAM, modelling may follow various methodologies, from scanning a wax-up (Cercon) to computer aided design (CAD- Lava, Digizon). The veneering of the high strength cores is performed using adapted conventional layering techniques with veneering leucite-ceramics.

Conventional cementation is allowed, ${ }^{1,2}$ but the digitizing process often demands a conical, low retentive preparation design. Therefore resin-based bonding systems are necessary to preserve suf- 
ficient strength of pressable and zirconia ceramics. . $^{3,4}$ To increase the surface roughness and facilitate bonding, air- abrasion with small particle $\mathrm{Al}_{2} \mathrm{O}_{3}$, pyrosil treatment, ${ }^{5}$ as well as tribochemical treatments ${ }^{6-7}$ have been utilized, however surface treatments such as hydrofluoric etching do not help to increase the roughness of the inert zirconia surface.

Interactions at the cement-FPD transition may also influence the bonding between cement and tooth. This transition is particularly sensitive, as insufficient sealing may allow the penetration of bacteria. Under clinical conditions, the continuing decrease of marginal adaptation may culminate in a loss of the restoration due to microleakage and resulting secondary caries. Generally, dentine pre-treatment includes acid, primer, adhesive and bonding agent. It is possible to combine two or all components in one solution. The optimal adhesive cementation interlocks strongly with the hybrid layer and tag formation, but the clinical application of the materials is time consuming and technically sensitive. Cements which show comparable adaptation but avoid the complex bonding procedures are therefore desirable. New, resin based bonding materials offer the option of bonding without separate tooth treatment. 8,9

The aim of this investigation was to evaluate the marginal adaptation of adhesively cemented CAD/CAM-made zirconia three-unit FPDs. Additionally, new self-adhesive resin cement without surface pre-treatment was evaluated on its marginal integrity in comparison to the other bonding systems. Metal-ceramic restorations with adhesive bonding and conventional cementation were used as controls.

\section{MATERIALS AND METHODS}

The roots of 96 freshly extracted human third molars were coated with a $1 \mathrm{~mm}$ thick layer of polyether impression material (Impregum: ESPE, Seefeld, Germanyl to simulate the resilience of the human periodontium. Two abutment teeth were embedded pair wise in PMMA resin (Palapress Vario: Kulzer, Wehrheim, Germanyl at a distance of $10 \mathrm{~mm}$ to represent a molar gap. The circular preparation of the teeth was performed with dia- mond burs resulting in $1 \mathrm{~mm}$ deep shoulders with finishing lines in dentine. Thirty-two three-unit posterior FPDs were made of the CAD-CAM System LAVA (3M Espe. Seefeld, Germany). For the fabrication of the 3-mol\% yttria tetragonal zirconia polycristal (Y-TZP), all-ceramic cores, gypsum models were optically scanned. The frameworks were designed with the computer and were milled in "green", pre-sintered ceramic condition to preserve time and milling tools. The final sintering process determines the physical properties and dimensions of the framework. The framework was veneered with the ceramic LAVA Ceram. Sixteen three unit metal FPDs (BioPontostar, Bego, Bremen, Germanyl were fabricated on master casts of class IV dental stone (Fuji Rock: GC-Corporation, Tokyo, Japanl and veneered with ceramic (Omega 900; Vita, Bad Säckingen, Germany). The cements and tooth pre-treatments used are listed in the Table 1. In the case of adhesive bonding, each abutment was light cured for $2 \times 40 \mathrm{sec}$ (Elipar Trilight, 3M Espe, Seefeld, Germany).

All abutment teeth were treated depending on the bonding system, and according to the manufacturer's instructions. The inner surfaces of the FPD retainers were tribochemically treated (Rocatec Soft, 3M Espe, G, Seefeld, Germanyl. 24 hours after luting, excessive cement was removed with diamond burs (Meisinger, Düsseldorf, Germany) and polishing instruments (Soflex-Scheiben: 3M Dental Products, St. Paul, USA). The FPDs were then placed in an artificial oral environment for thermal cycling and mechanical loading (TCML). Thermal cycling was performed rinsing the FPDs with bi-distilled water of $5^{\circ} \mathrm{C}$ and $55^{\circ} \mathrm{C}$, respectively. Each temperature, $5^{\circ} \mathrm{C}$ and $55^{\circ} \mathrm{C}$ was kept for two minutes. Simultaneously, the FPDs were centrally loaded with a human antagonist. A force of $50 \mathrm{~N}$ was applied $1.2 \times 10^{6}$ times with at a frequency of $1.6 \mathrm{~Hz}$. Artificial aging was performed according to Krejci to simulate 5 years of intraoral service. ${ }^{10-11}$

For the semi-quantitative analysis of the marginal adaptation, both the cement-tooth and the cement-FPD-interfaces were examined using a scanning electron microscope (SEM), Stereoscan 240 (Cambridge Instruments, Nußloch, Germany; magnification: 200x-800x; working distance 18

Table 1. Materials and manufacturers.

\begin{tabular}{|c|c|c|c|c|}
\hline FPD material & Cement & Bonding & Manufacturer & Cement type \\
\hline \multirow{4}{*}{$\begin{array}{l}\text { All-ceramic } \\
\text { (LAVA/Lava Ceram, 3M Espe, G) }\end{array}$} & Variolink 2 & Syntac Classic & (Ivoclar-Vivadent, FL) & \multirow{3}{*}{ Resin-cement } \\
\hline & Panavia F & ED & (Kuraray, J) & \\
\hline & Compolute & EBS Multi & (3M Espe, G) & \\
\hline & RelyX Unicem & without & (3M Espe, G) & \multirow{2}{*}{$\begin{array}{l}\text { Universal } \\
\text { resin- cement }\end{array}$} \\
\hline \multirow{2}{*}{$\begin{array}{l}\text { Metal-ceramic } \\
\text { BioPontostar (Bego, G)/ Vita } \\
\text { Omega (Vita, G) }\end{array}$} & RelyX Unicem & without & (3M Espe, G) & \\
\hline & Harvard & without & (Harvard, G) & Zn-phosphate \\
\hline
\end{tabular}


mm). Replicas (Epoxy VP 1031, Vivadent, Schaan, FL, Schaan, Liechtenstein) of the cervical margins of both abutment teeth were made before and after TCML using impressions (Permadyne, 3M Espe; Seefeld, Germanyl and special resin trays (diameter $50 \mathrm{~mm}$ ). The replicas were sputtered coated with a thin layer of gold to ensure conductivity and hinder electricity charge during SEM analysis. The cervical margins, including the whole preparation line from oral over approximal to buccal were investigated and the percentage of "perfect margin" (smooth transition, no interruption of continuity) and "marginal gap" (separation of the components because of adhesive and/or cohesive failure) were determined ${ }^{12}$ (example see. Figure 1). To improve reproducibility, one investigator evaluated all specimens. Figure 2 demonstrates the FPD design, shoulder preparation and the area, where the analysis was performed.

For the dye penetration test, the FPDs were incubated in a basic fuchsine solution $(0.5 \%)$ for 16 hours at $37^{\circ} \mathrm{C}$. Then the abutment teeth were cut into slices of about $500 \mu \mathrm{m}$ thickness using a water-cooled cutter (Leica, SP1600, Bensheim, Germanyl. Immediately after cutting, the slices were digitized using the light microscope Stemi SV8 (Zeiss, Oberkochen, Germany) to avoid artifacts because of bleaching or water penetration.

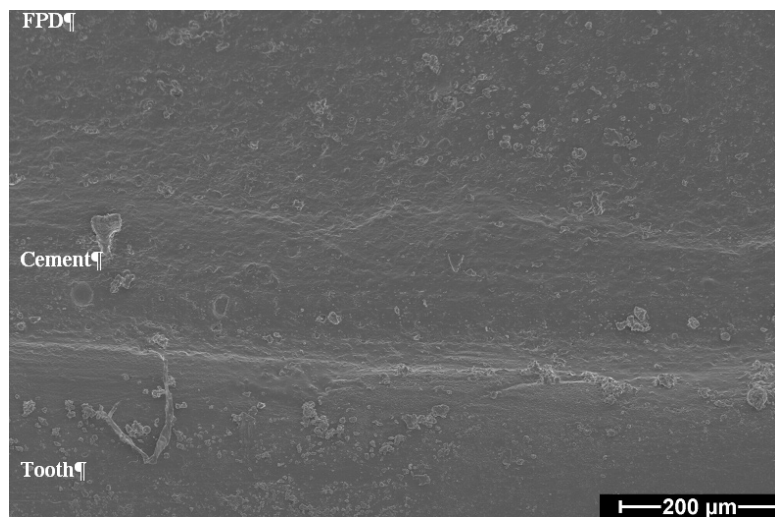

The depth of the dye penetration l= intrusion of the fuchsine solution) and the depth of the shoulder preparation were determined using an image analysing system (Optimas 6.1, Media Cybernetics, Silver Spring, MD, USA). Percentage microleakage is defined as the percentage of dye penetration in proportion to the depth of the preparation. Figure 3 shows a photo of the dye penetration, including the principle analysis.

Median and 25/75\%-percentiles were calculated. Statistical analysis was performed using the Mann-Whitney-U-Test and Kruskal-Wallis-Test for non-normal distributed values (SPSS/PC+ 5.0, SPSS, USA)..$^{13}$ The level of significance was set to $P=0.05$.

\section{RESULTS}

\section{Marginal adaptation}

At the cement-FPD interface, the cement systems showed greater than $95 \%$ perfect margins except for Lava with Variolink 2/Syntac classic, which had a significantly lower $(P=0.000)$, approximately $65 \%$ perfect margin. After TCML, marginal integrity decreased for all materials by a maximum of $5 \%$ for Lava with Variolink 2/Syntac classic. The control BioPontostar/RelyX Unicem showed a perfect margin of $98 \%$ which deteriorated to $96 \%$ after TCML. BioPontostar/Harvard had values of $95 \%$

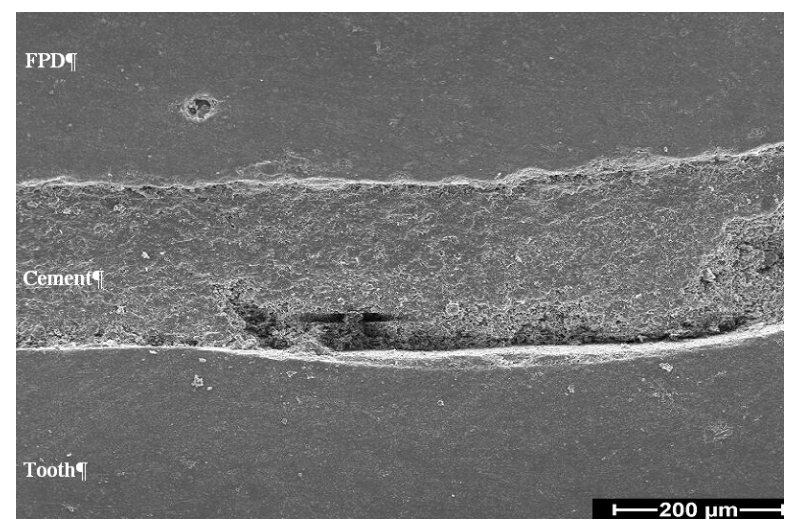

Figure 1. Section of the cervical margin indicating the criteria for the marginal evaluation (SEM picture, x400, working distance $18 \mathrm{~mm}$ l left: perfect margin right: marginal gap between cement and tooth.

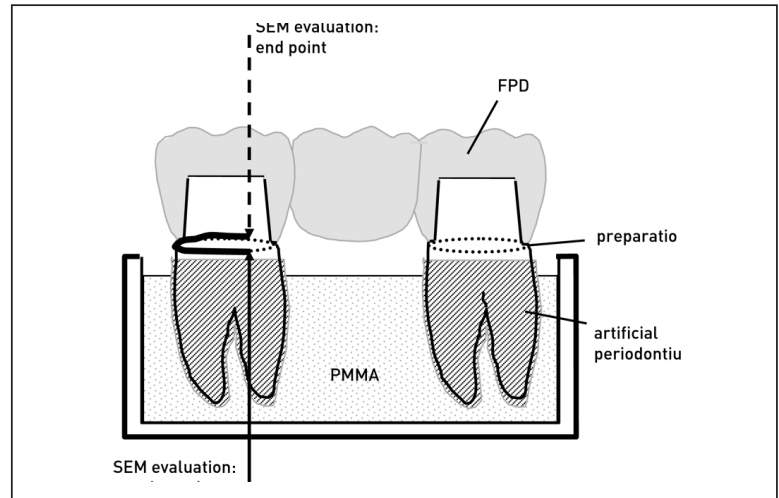

Figure 2. FPD design with $1 \mathrm{~mm}$ dentin shoulder preparation; arrows indicate the starting and end of the evaluation of the marginal quality.

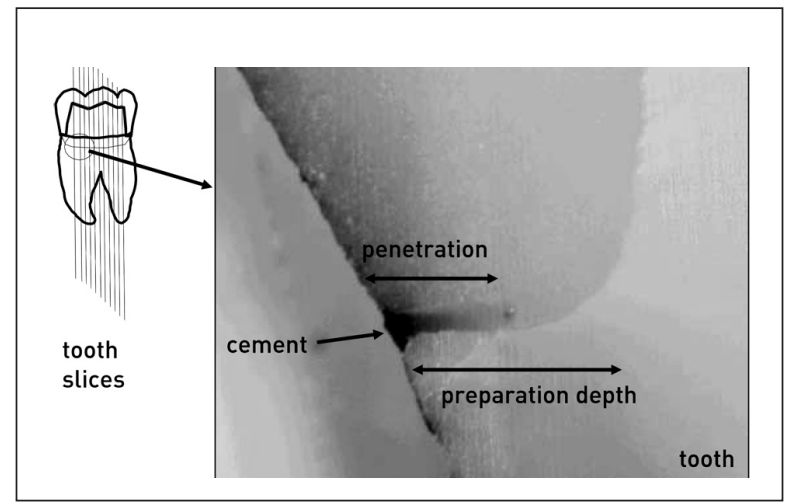

Figure 3. Dye penetration photograph with schematic diagram of the microleakage analysis between FPD, cement and tooth. 
before, and $94 \%$ after TCML (Figure 4, above).

At the cement-tooth interface, over $95 \%$ perfect margins were observed for Lava with Panavia/ED, Compolute/EBS multi and RelyX Unicem. No decrease was found after TCML. For Variolink $2 /$ Syntac classic the integrity was $70 \%$, which decreased significantly $(P=.042)$ by $10 \%$ after TCML. BioPontostar with RelyX Unicem showed results of $97 \%$ perfect margin before and after TCML. For BioPontostar with Harvard, 95\% perfect margins were found. The values decreased significantly $(P=.018)$ to $93 \%$ after TCML (Figure 4, below).

The standard deviation of the marginal SEM analysis was $6.0 \%$ and the standard error $0.3 \%$. Statistical differences were indicated in Figure 4.

\section{Dye penetration}

Lava with Panavia/ED, Compolute/EBS multi and RelyX Unicem showed penetration values lower than $5 \%$ at both cement-tooth and cement-FPD interfaces. For Variolink 2/Syntac classic, a significantly higher penetration (75\%) was found at the cement-tooth interface $(P=.000)$ and almost $100 \%$ $(P=.000)$ at the cement-FPD interface. For the BioPontostar/RelyX Unicem FPDs, 15\% leakage could be determined at the cement-tooth transi-
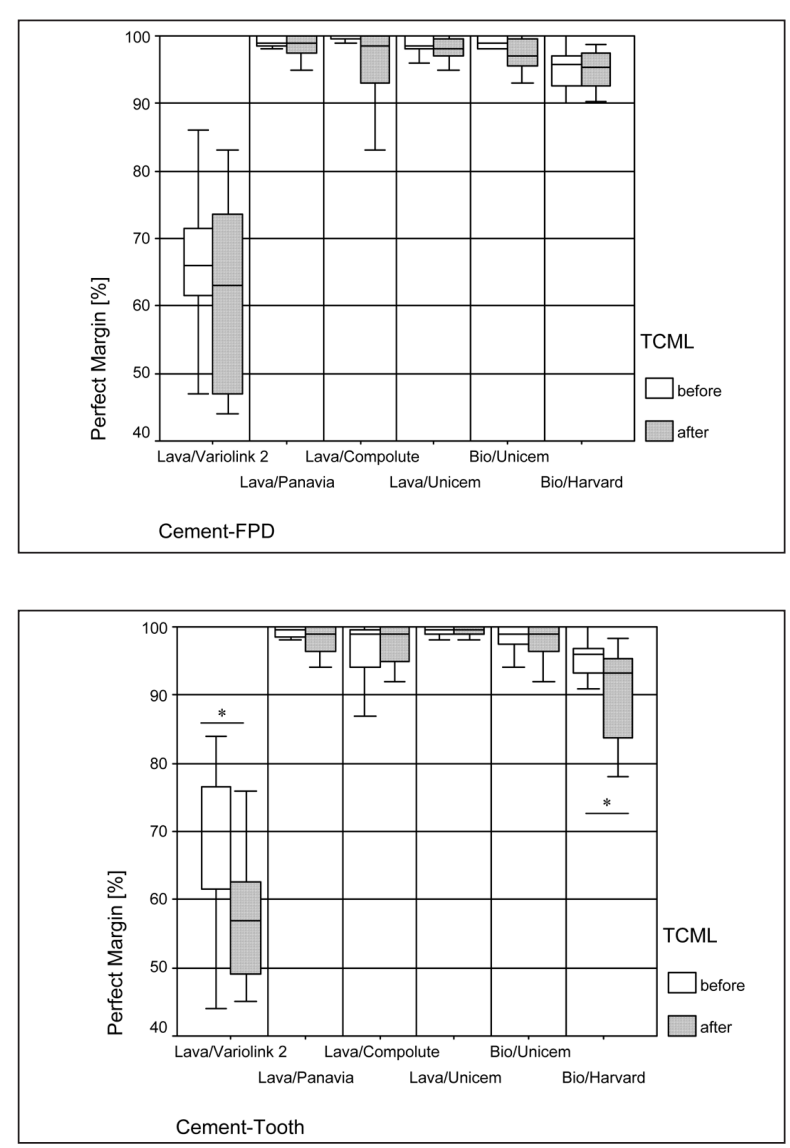

Figure 4. SEM marginal adaptation before and after aging: criterion "perfect margin [\%]"; above: transition cement-FPD, below: transition cement-tooth (median, 25\% / 75\% percentiles, min, max); statistically significant differences are indicated by tion and about $6 \%$ at the cement-FPD interface. With $100 \%$ leakage at the cement-tooth transition and $0 \%$ leakage at the cement-FPD transition, the BioPontostar/Harvard FPDs showed significantly worse results (Figure 5).

The standard deviation of measurement was $28.6 \%$ and the standard error $1.5 \%$. Statistical differences were indicated in Figure 5.

\section{DISCUSSION}

Scanning electron microscopy (SEM) evaluation is an established and reproducible procedure for determining the cervical marginal adaptation. It is based on comparable conditions and semi-quantitative analysis. ${ }^{14}$ Of course, accuracy of impression taking or replica fabrication limits the value of such procedures. The deviation of SEM analysis showed, that the semi-quantitative analysis, which was performed by one examiner, improved subjectivity and error rate. Microleakage tests with dye solution supplement the superficial marginal SEM evaluation with internal bond penetration results. The high deviations of the microleakage measurements may be a result of individual differences in tooth tissue combined with the variability of bonding quality. Microleakage and SEM results may differ, even when main parts of the circular margin are sealed ("perfect margin"): dye solution may penetrate through remaining small gaps or even the cement may chemically absorb and forward the basic fuchsine solution. Only a sealed hard tooth structure completely may hinder microleakage.

In SEM evaluation and microleakage tests, the marginal adaptation showed low penetration and a high percentage bonding between adhesive and LAVA FPDs. Only for Variolink2/Syntac classic was insufficient results found. In contrast to glassceramics, the inert zirconia ceramic can not be etched for pre-treating and activating the surface.

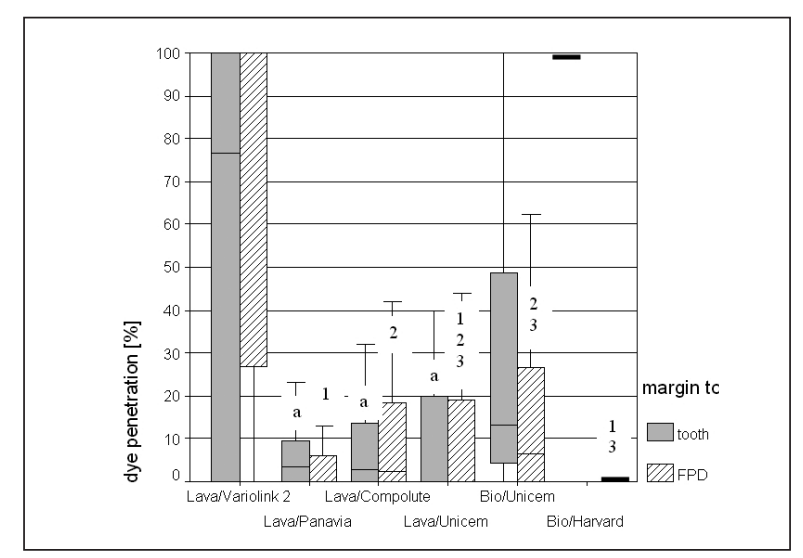

Figure 5. Dye penetration [\%] after artificial aging (median, $25 \%$ / 75\% percentiles, min, max); identical letters/numbers identify statistically identical results. 
The applied treatment with tribochemical methods allowed a successful bonding between cement and FPD, but the bond strength between zirconia and cement requires further investigations, e.g., using tensile tests.

Clinically applied resin-based bonding systems, such as Panavia/ED or Compolute/EBS, 15-16 as well as the new system RelyX Unicem without tooth treatment, showed high marginal integrity between cement and tooth. The bonding conditions between resin cement and tooth were determined by the condition of the dentin smear layer and the etching procedure. Highest bonding results were obtained by dissolving or removing the smear layer and the formation of resin tags, ${ }^{17-18}$ like provided by Panavia/ED and Variolink/Syntac. RelyX Unicem without pre-treatment showed a marginal integrity comparable to the resin cements. According to the manufacturer, the resin-based RelyX Unicem is a smear layer modifying, self-adhesive, dual curing cement. It is based on methacrylated phosphoric acid esters with several cement reactions during setting. The functional groups of the modified acrylates initiate a neutralization reaction with the basic salt and the tooth apatite. In this step, water is released which may contribute to the hydrophilicity and adaptation to dentin. Without tooth pre-treatment, the smear layer remains on the tooth surface ${ }^{8-9}$ and hinders the creation of resin tags in the dentin tubuli. An incomplete penetration of the smear-layer with bonding agent or cement would weaken the resulting bond. An adhesive failure may be located between bonding and hybrid layer or between hybrid layer and sound dentin. ${ }^{19}$ Therefore, systems with modified smear layers often show low bond strength values, ${ }^{20}$ but for RelyX Unicem a bond strength similar to the resin cements is reported. ${ }^{21}$ In contrast to the other resin cements, the marginal integrity for LAVA/ Variolink2/Syntac was significantly lower at the transition between cement and tooth. Similar high dye penetration was found in previous investigations. ${ }^{8}$ The low marginal adaptation and the high amount of dye penetration, indicate a disturbed chemical reaction of the bonding system or cement. A reason may be an inadequate activation of the bonding system due to opaque, light blocking all-ceramic core.

Regarding the metal-ceramic FPD controls, the bonding with Unicem and conventional Zincphosphate showed comparable marginal adaptation. The high penetration results for Harvard cement at the cement-tooth interface are remarkable. Further microleakage investigations on Zinc-phosphate cement remain to be tested with alternative dye solutions, such as methanol-blue.

\section{CONCLUSIONS}

Under the conditions of this study, zirconia FPDs showed good to sufficient marginal integrity in combination with Panavia/ED, Compolute/EBS and RelyX Unicem. Restrictions had to be made with the combination Variolink2/Syntac. The adhesive bonding of zirconia restorations in combination with a tribochemical treatment ${ }^{22}$ or air abrading with small particle $\mathrm{Al}_{2} \mathrm{O}_{3}$ is a promising application, but the results are dependent on the choice of cement ${ }^{23}$ and bonding agent. The results suggest that RelyX Unicem without tooth treatment is comparable to an adhesive cementation with regard to marginal integrity and mircoleakage. The bonding mechanisms of this cement should be investigated further.

\section{REFERENCES}

1. Blatz MB, Sadan A, Kern M. Resin ceramic bonding: a review of the literature. J Prosthet Dent 2003;89:268-274.

2. Tinschert J, Natt G, Mautsch W, Augthun M, Spiekermann $H$. Fracture resistance of lithium disilsicate-, alumin-, and zirkonia-based three-unit fixed partial dentures: a laboratory study. Int J Prosthodont 2001;14:231-238.

3. Jensen ME, Sheth JJ, Tolliver D. Etched-porcelain resinbonded full-veneer crowns: in vitro fracture resistance. Compendium 1989;10:336-338,340-1,344-7.

4. Behr M, Rosentritt M, Mangelkramer M, Handel G. The influence of different cements on the fracture resistance and marginal adaptation of all-ceramic and fiber-reinforced crowns. Int J Prosthodont 2003;16:538-542.

5. Janda R, Roulet JF, Wulf M, Tiller HJ. A new adhesive technology for all-ceramics. Dent Mater 2003;19:567-573.

6. Bulot D, Sadan A, Burgess J.O., Bletz M.B. Bond strength of a self-adhesive universal resin cement to Lava zirkonia after two surface treatments. J Dent Res 2003; 81: Abstr. No 578.

7. Edelhoff D, Sorensen JA. Retention of Selected Core Materials to Zirconia Posts. Oper Dent 2002;27:455-461.

8. Rosentritt M, Behr M, Lang R, Handel G. Influence of Cement Type on the Marginal Adaptation of All-Ceramic MOD Inlays. Dent Mater 2004;20;463-469.

9. Behr M, Rosentritt M, Regnet T, Lang R, Handel G. Marginal adaptation in dentin of a self-adhesive universal resin cement compared with well-tried systems. Dent Mater 2004; 20:191-197.

10. Krejci I, Lutz F. In-vitro test results of the evaluation of dental restoration systems. Correlation with in-vivo results. Schweiz Monatsschr Zahnmed 1990;100:1445-1449.

11. Rosentritt M, Leibrock A, Lang R, Scharnagl M, Behr M, Handel G: Regensburger masticator. Materials Testing 1997; 3:77-80.

12. Schmalz G, Federlin M, Reich E. Effect of dimension of luting space and luting composite on marginal adaptation of class II ceramic inlays. J Prosthet Dent 1995;73:392-399.

13. Brosius G, Brosius F. SPSS Base System und Professional Statistics, 1st edn. Bonn, Albany: International Thomson Publishing, 1995.

14. Roulet JF, Reich T, Blunck U, Noack M. Quantitative margin analysis in the scanning electron microscopy. Scanning Microscopy 1989;3:147-159

15. Van-Dijken JW. Resin-modified glass ionomer cement and self-cured resin composite luted ceramic inlays. A 5-year clinical evaluation. Dent Mater 2003;19:670-674.

16. Felden A, Schmalz G, Hiller KA. Retrospective clinical study and survival analysis on partial ceramic crowns: results up to 7 years. Clin Oral Invest 2000;4:199-205.

17. Koibuchi, Yasuda N, Nakabayashi N. Bonding to dentin with 
a self-etching primer: the effect of smear layers. Dent Mater 2001;17:122-126.

18. Shinchi MJ, Soma K, Nakabayashi N. The effect of phosphoric acid concentration on resin tag length and bond strength of a photo-cured resin to acid-etched enamel. Dent Mater 2000;16:324-329.

19. Kiyomura M. Bonding strength to bovine dentin with 4Meta/Mma-TBB resin. Long-term stability and influence of water. J Jpn Dent Mater 1987;6:860-872.

20. Koibuchi H, Yasuda, N, Nakabayashi N. Bonding to dentin with a self-etching primer: the effect of smear layers. Dent Mater 2001;17:122-126.

21. Irie M, Suzuki K, Windmüller B. Effect of one-day storage on marginal gap of composite inlays. J Dent Res 2002;81: Abstr. No. 3365.

22. Sahafi A, Peutzfeldt A, Asmussen E, Gotfredsen K. Bond strength of resin cement to dentin and to surface-treated posts of titanium alloy, glass fiber, and zirconia. $J$ Adhes Dent 2003;5:153-162.

23. Wegner SM, Kern M. Long-term resin bond strength to zirconia ceramic. J Adhes Dent 2000;2:139-147. 